\title{
ARTICLES
}

\section{First-principles determination of the effects of boron and sulfur on the ideal cleavage fracture in $\mathrm{Ni}_{3} \mathrm{Al}$}

\author{
Sheng N. Sun and Nicholas Kioussis \\ Department of Physics and Astronomy, California State University, Northridge, California 91330-8268 \\ Mikael Ciftan \\ Physics Division, U.S. Army Research Office, Research Triangle Park, North Carolina 27709-2211
}

(Received 21 November 1995)

\begin{abstract}
The effects of boron and sulfur impurities on the ideal cleavage fracture properties of $\mathrm{Ni}_{3} \mathrm{Al}$ under tensile stress are investigated using the full-potential linear-muffin-tin-orbital total-energy method, with a repeated slab arrangement of atoms simulating an isolated cleavage plane. Results for the stress-strain relationship, ideal cleavage energies, ideal yield stress and strains with and without impurities are presented, and the electronic mechanism underlying the contrasting effects of boron and sulfur impurities on the ideal cleavage of $\mathrm{Ni}_{3} \mathrm{Al}$ is elucidated. [S0163-1829(96)03329-2]
\end{abstract}

The $L 1_{2}$-type ordered nickel aluminide, $\mathrm{Ni}_{3} \mathrm{Al}$, exhibits unique mechanical properties that make it attractive for structural applications at elevated temperatures. ${ }^{1}$ These are its high melting temperature, low density, resistance to oxidation, ${ }^{2}$ and the increase of yield stress with increasing temperature, ${ }^{3}$ in contrast to conventional compounds or disordered alloys. However, as with many other intermetallics, an inherent drawback to using polycrystalline ordered stoichiometric $\mathrm{Ni}_{3} \mathrm{Al}$ alloys as a structural material is the tendency to undergo brittle intergranular fracture, ${ }^{4}$ even though single crystals of $\mathrm{Ni}_{3} \mathrm{Al}$ are highly ductile. Weak grainboundary cohesion and low cleavage strength have been suggested $^{5}$ to be two of the main causes of brittleness in polycrystalline intermetallic systems. Microalloying studies have shown that doping with boron, which strongly segregates to the grain boundaries, can significantly improve the ductility of polycrystalline $\mathrm{Ni}_{3} \mathrm{Al},{ }^{5}$ whereas sulfur reduces ductility and causes embrittlement. ${ }^{6}$

It is well known that the complex and varied microstructures of grain boundaries play central roles in determining the mechanical behavior of these systems. ${ }^{7}$ Studies have been underway to "design grain boundaries" 8 in order to affect the thermomechanical behavior of these alloys. The manner in which electronic charge becomes localized or spread among neighboring atoms will make a significant contribution to their thermomechanical behavior. Thus, the region between adjacent grains, the grain-boundary domain, can be very sharp or extended spatially with simple or complex local structures tied to the chemical thermodynamics of these systems creating stable or metastable structures out of the local chemistry. ${ }^{7}$ The fracture mode of brittle intermetallics is either cleavage, i.e., fracture along specific crystallographic planes in either a single-crystal or polycrystalline sample, or intergranular, i.e., fracture along the grain boundaries in a polycrystalline sample. ${ }^{9}$ Addition of atomic impurities which segregate to the grain-boundary domain, signifi- cantly modifies the complex behavior of fracture that propagates along or through the grains. Thus, boron ${ }^{5}$ has been known to suppress intergranular fracture in contrast to sulfur $^{6}$ that promotes it. More recent experiments ${ }^{10}$ have shown that boron also improves the ductility of single $\mathrm{Ni}_{3} \mathrm{Al}$ crystals, suggesting that a "bulk effect" should be considered in addition to the grain-boundary strengthening of boron when explaining the improvement in ductility of polycrystalline $\mathrm{Ni}_{3} \mathrm{Al}$ due to $\mathrm{B}$ additions.

While significant progress is currently being made in atomistic simulation calculations of fracture processes, ${ }^{11}$ inclusion of realistic materials characteristics (grain boundaries, dislocations, etc.) in first-principles electronic-structure calculations remains very difficult. Since, in the case of the $\mathrm{Ni}_{3} \mathrm{Al}$, the crystalline structure of the grains remains ordered very close to the boundary plane, producing a relatively sharp grain boundary, with or without boron addition, ${ }^{7}$ it is reasonable to expect that a first-principles study of the bonding characteristics of atoms in a supercell with and without boron and/or sulfur impurities will provide insight into the cohesive forces that control cleavage failure. The next level of study would then be to include the relaxation of the crystalline atomic positions (emulating the partial disorder that has been revealed experimentally very close to and at the grain boundary), and to determine the effect of such perturbations on cleavage properties, some of which have proved useful in phenomenological models of fracture, but can only be obtained from first-principles investigations. Among these are the upper bound of tensile yield stresses, the "ideal yield stress," and the ideal cleavage energy (which is equal to the total surface energy $\gamma_{s}$ of the two cleaved surface planes), a quantity which appears in the phenomenological theory recently proposed by Rice ${ }^{12}$ for determining the intrinsic ductile versus brittle behavior of materials. Rice proposed that a simple rule to measure the brittle and/or ductile behavior of materials is the ratio $\gamma_{u s} / \gamma_{s}$, which determines the competi- 


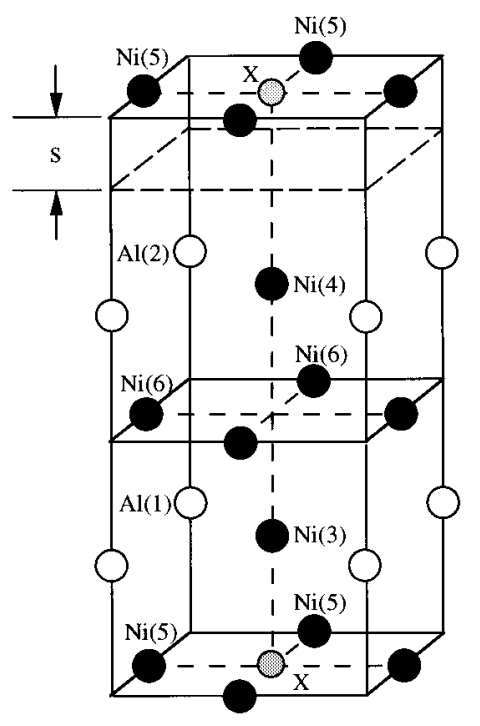

FIG. 1. Supercell geometry used in the cleavage calculation. The filled, empty, and gray shaded circles represent sites for $\mathrm{Ni}, \mathrm{Al}$, and impurity ( $X$ stands for boron or sulfur) atoms, respectively. Atoms of different symmetry are distinguished by a type number.

tion between dislocation emission from a crack tip and crack cleavage. Dislocation nucleation is characterized by the unstable stacking fault energy $\gamma_{u s}$, which is the maximum energy barrier encountered in sliding one half of a crystal relative to another along a slip plane.

In an attempt to determine these parameters and develop a more fundamental understanding of fracture phenomena, recent theoretical efforts have applied first-principles electronic-structure calculations to study the atomic-level factors that underlie the effect of impurities on grainboundary cohesion and intergranular fracture. ${ }^{13-18}$ Using quantum-mechanical methods, Messmer and Briant ${ }^{13}$ modeled Ni or Fe metal clusters coordinating different impurities. The contrasting behaviors of the added boron and sulfur impurities have been interpreted ${ }^{13}$ as caused by modification of the local electronic structure induced by these defects near and within the grain boundary in specific ways; "embrittling elements," such as sulfur, draw charge from neighboring metal atoms onto themselves, thereby weakening the metalmetal bond charge network, whereas "cohesive enhancers" such as boron, do not draw charge from their neighboring metal atoms, forming homopolar bonds, and maintain the cohesiveness of the metal-metal atom charge network. ${ }^{13}$ The electronic-structure cluster calculations of Eberhart and Vvedensky ${ }^{14}$ have shown that the appearance of localized grain-boundary electronic states in $\mathrm{Ni}_{3} \mathrm{Al}$, with considerably less directional charge distribution than that found in the parent crystal, provides an indication of intergranular fracture. In a later paper, ${ }^{15}$ it was further suggested that boron possesses an electronic configuration which, due to symmetry considerations, can stabilize the noncrystallographic bonding geometries found near grain boundaries. Electronicstructure calculations ${ }^{16}$ have also been used to investigate the effect of charge redistribution on the interfacial strength during the process of straining. First-principles atomic-cluster calculations ${ }^{17}$ of the total energy and the gradient forces have shown that boron (sulfur) interstitial impurities in a nickel

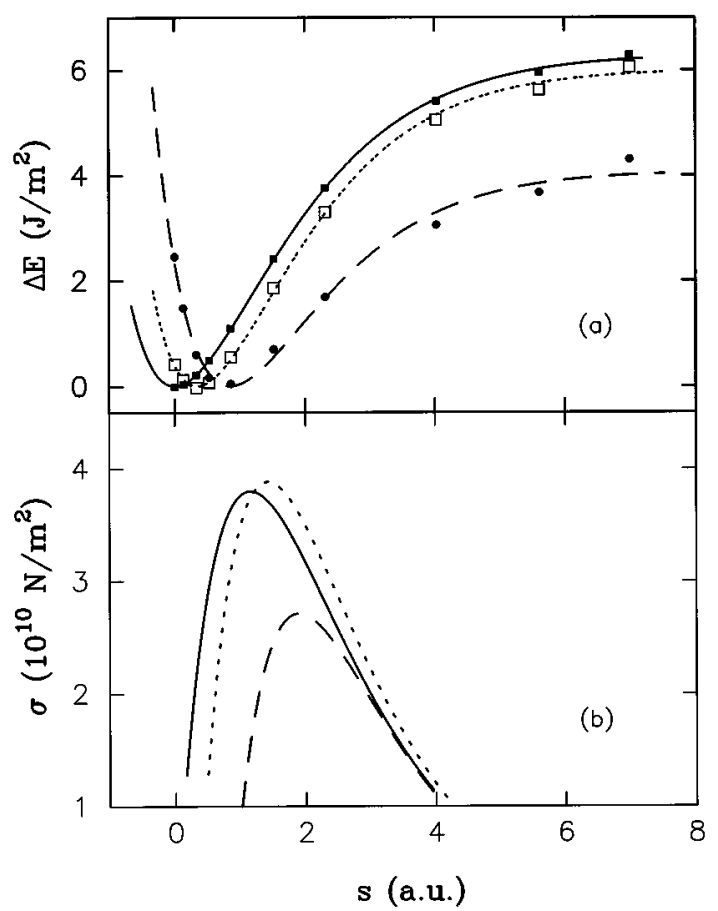

FIG. 2. (a) The calculated total-energy difference per unit area, $\Delta E$, versus cleavage separation for $\mathrm{Ni}_{3} \mathrm{Al}$ (solid squares), $\mathrm{Ni}_{3} \mathrm{AlB}_{1 / 2}$ (open squares), and $\mathrm{Ni}_{3} \mathrm{AlS}_{1 / 2}$ (solid circles). The solid, short- and long-dashed lines are the least-squares fits. (b) The cleavage stress versus cleavage separation for $\mathrm{Ni}_{3} \mathrm{Al}$ (solid line), $\mathrm{Ni}_{3} \mathrm{AlB}_{1 / 2}$ (shortdashed line), and $\mathrm{Ni}_{3} \mathrm{AlS}_{1 / 2}$ (long-dashed line).

cluster increase (decrease) the maximum sustainable restoring force in the cluster. More recently, Wu, Freeman, and Olson $^{18}$ have carried out full-potential linear-augmented plane-wave calculations to determine the difference in interfacial energy associated with the embrittling potency of boron and phosphorus impurities in iron.

In the present study, we take a different approach to the understanding of role segregation plays in cohesion, by investigating the effect of impurities on the ideal cleavage properties of single $\mathrm{Ni}_{3} \mathrm{Al}$ crystals. Using highly accurate total-energy self-consistent electronic-structure calculations, based on the full-potential linear-muffin-tin-orbital (FLMTO) method, ${ }^{19}$ we have investigated the effect of impurities on the ideal cleavage properties (cleavage energy and yield stress) of $\mathrm{Ni}_{3} \mathrm{Al}$. Though the method, as used here, is capable of dealing with very open systems, such as surfaces,${ }^{20}$ it is not constrained to surface situations, so that a continuous transition from a bulk, closely packed arrange-

TABLE I. Calculated values for the ideal cleavage energy, $G_{e}$, the maximum tensile stress $\sigma_{\max }$, and the ideal strain $\epsilon$ corresponding to the maximum tensile stress, for fracture cleavage of the (001) planes of $\mathrm{Ni}_{3} \mathrm{Al}, \mathrm{Ni}_{3} \mathrm{AlB}_{1 / 2}$, and $\mathrm{Ni}_{3} \mathrm{AlS}_{1 / 2}$.

\begin{tabular}{lccc}
\hline \hline Compound & $\begin{array}{c}G_{e} \\
\left(\mathrm{~J} \mathrm{~m}^{-2}\right)\end{array}$ & $\begin{array}{c}\sigma_{\max } \\
\left(10^{10} \mathrm{~N} \mathrm{~m}^{-2}\right)\end{array}$ & $\epsilon$ \\
\hline $\mathrm{Ni}_{3} \mathrm{Al}$ & 6.31 & 3.80 & $35 \%$ \\
$\mathrm{Ni}_{3} \mathrm{AlB}_{1 / 2}$ & 6.02 & 3.89 & $30 \%$ \\
$\mathrm{Ni}_{3} \mathrm{AlS}_{1 / 2}$ & 4.08 & 2.71 & $25 \%$ \\
\hline \hline
\end{tabular}




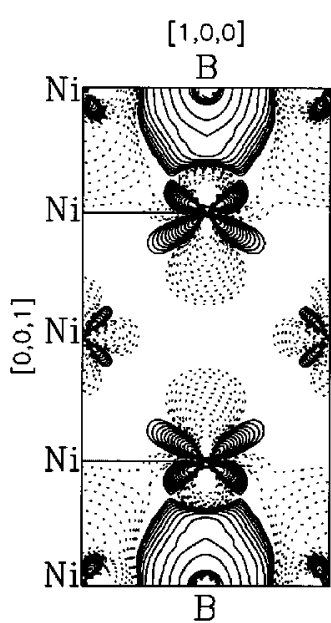

$(s=0.0)$

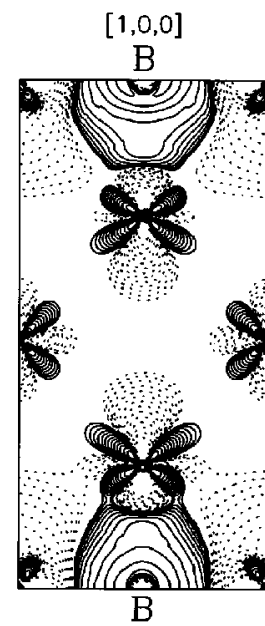

$(s=0.05 a)$

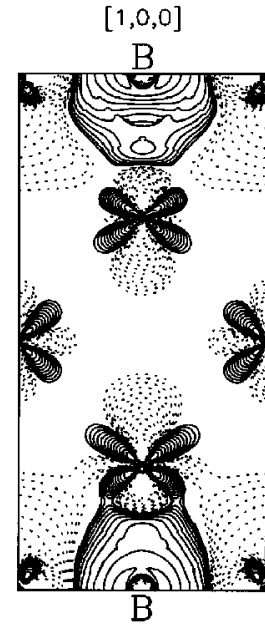

$(s=0.080)$

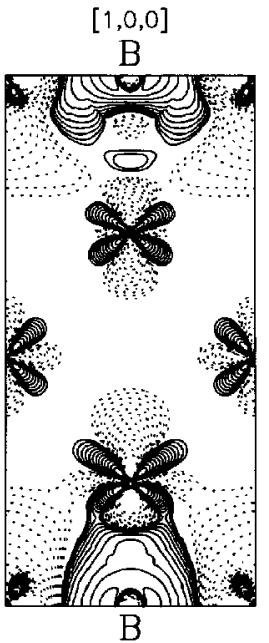

$(s=0.13 a)$
FIG. 3. Boron-induced charge density on the (200) plane for various slab separation ratios $s / a$. Solid (dotted) contours denote contours of increased (decreased) charge density. Contours start from $\pm 8.0 \times 10^{-4} \mathrm{e} /(\text { a.u. })^{3}$ and increase successively by a factor of root 2 . ment of atoms to an open arrangement, as when cleavage planes are created, can also be treated. In the FLMTO method, ${ }^{19}$ no shape approximation need be made to the potential and the charge density (the iteration process to selfconsistency does not require a spherical averaging of the potential and the charge density). Thus, these calculations can provide accurate information on the tensorial/directional properties of the bonding forces across and parallel to the cleavage plane in terms of the anisotropy of the detailed induced bonding charge characteristics.

The creation of a cleavage plane is achieved in this calculation by using a repeated slab construction. Each cell, shown in Fig. 1, contains one "slab" and each slab consists of four layers of (001) planes of $\mathrm{Ni}_{3} \mathrm{Al}$. Inequivalent atoms in the cell are denoted by numerical labels (enclosed in parenthesis), depending on their point-group symmetry. The simulation of the separation of two neighboring planes (cleaved surfaces) is accomplished by varying the separation, $s$, between the slabs. For $s=0$ we have bulk $\mathrm{Ni}_{3} \mathrm{Al}$, and as $s$ is increased the slab separation increases. Thus, we obtain a repeated slab configuration of isolated surfaces at large separations. The impurity is placed at the octahedral interstitial site labeled by $X$, where the impurity has four $\mathrm{Ni}(5)$, one
$\mathrm{Ni}(4)$, and one $\mathrm{Ni}(3)$ nearest neighbors. Such an arrangement corresponds to an impurity concentration of 11.1 at. \%. In order to minimize the effect of interactions between impurities within the (001) planes, we are currently carrying out electronic-structure calculations employing a slab twice as large as that in Fig. 1, with an impurity concentration of 5.88 at. $\%$. This will allow us to investigate the effect of impurity concentration on cleavage properties. Even though the impurity concentration in the calculations is high relative to the experimental values, ${ }^{1}$ we believe that these calculations serve as a first step in understanding the effects of impurities on the cleavage properties (cleavage energies, ideal yield stress, etc.) in $\mathrm{Ni}_{3} \mathrm{Al}$.

In Fig. 2(a) we have plotted the difference in total energy per unit area, $\Delta E$, of the four-layer unit cell versus the separation ( $s$ in Fig. 1) between neighboring slabs, for the pure $\mathrm{Ni}_{3} \mathrm{Al}$ system (solid squares), the $\mathrm{Ni}_{3} \mathrm{AlB}_{1 / 2}$ system (open squares), and the $\mathrm{Ni}_{3} \mathrm{AlS}_{1 / 2}$ system (solid circles). Here, $\Delta E$ refers to the total energy of the slab at separation $s$ minus the total energy at separation $s_{0}$, where $s_{0}$ represents the slab separation at the minimum of the total energy. Atomic relaxation between the (001) layers within the slab was ignored and the lattice constant was held fixed at the value of 3.568 $\AA$ for pure $\mathrm{Ni}_{3} \mathrm{Al}^{21}$ The difference in total energy $\Delta E$ con-
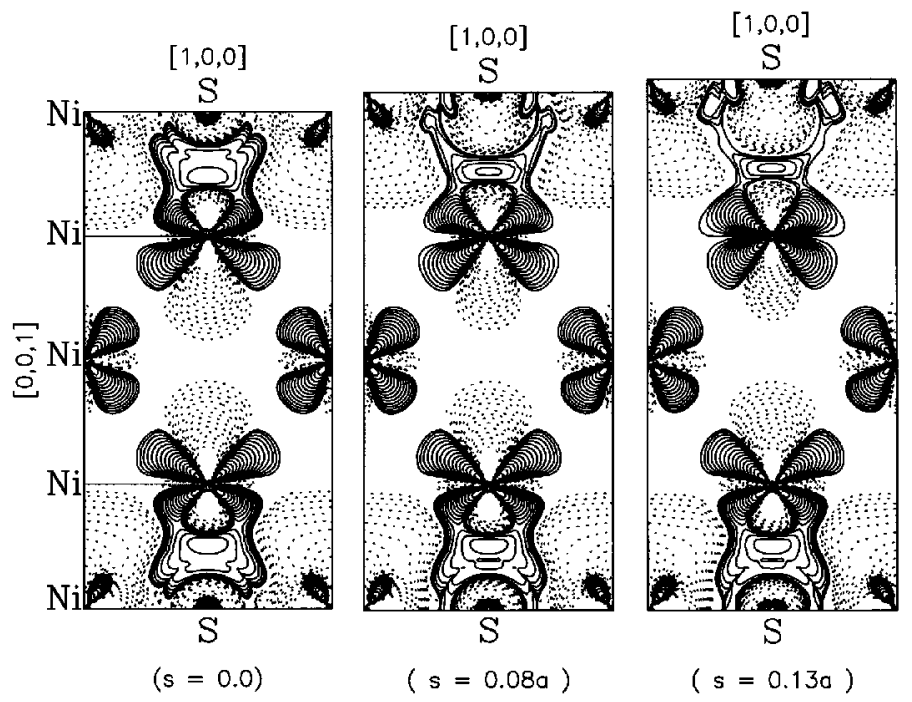

$(s=0.13 a)$

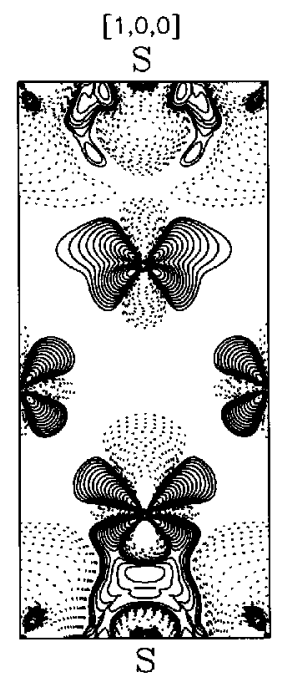

$(s=0.23 a)$
FIG. 4. Sulfur-induced charge density on the (200) plane for various slab separation ratios $s / a$. Solid (dotted) contours denote contours of increased (decreased) charge density. Contours start from $\pm 8.0 \times 10^{-4} \mathrm{e} /(\text { a.u. })^{3}$, and increase successively by a factor of root 2 . 
DOS of $\mathrm{Ni}_{3} \mathrm{AlB}_{1 / 2}$

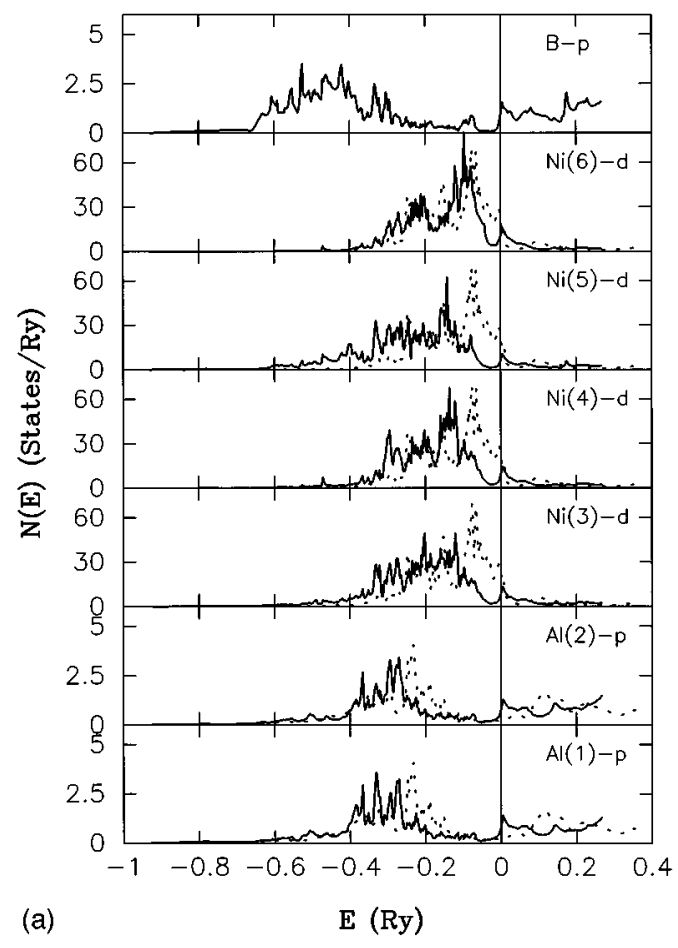

DOS of $\mathrm{Ni}_{3} \mathrm{AlS}_{1 / 2}$

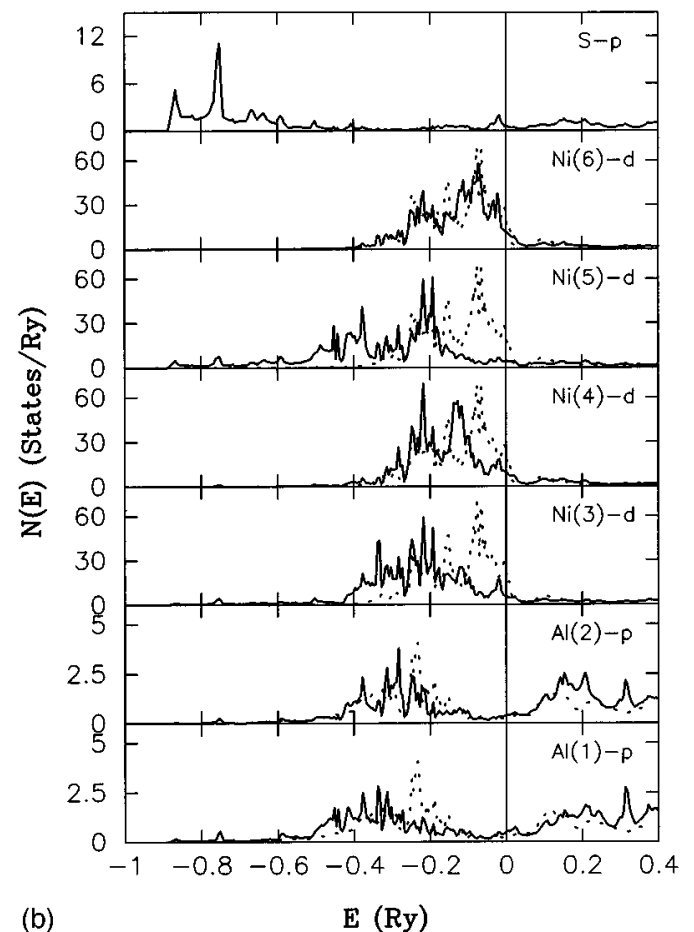

FIG. 5. Comparison of the $l$ - and site-projected DOS of $\mathrm{Ni}_{3} \mathrm{Al} X_{1 / 2}$ (solid lines) with that of the pure system $\mathrm{Ni}_{3} \mathrm{Al}$ (dotted lines). (a) DOS for boron-doped system $(X=\mathrm{B})$ with slab separation $s_{0}$ and (b) DOS for sulfur-doped system $(X=\mathrm{S})$ with slab separation $s_{0}$.

verges to the ideal cleavage energy (Griffith energy) as the separation between neighboring slabs increases (the ideal cleavage energy is the total surface energy of the two cleaved surface planes). We find that sulfur reduces the ideal cleavage energy of the pure $\mathrm{Ni}_{3} \mathrm{Al}$ system by $35 \%$, while boron reduces it only by $5 \%$. This small reduction in total energy and the small increase in the maximum tensile stress of $\mathrm{Ni}_{3} \mathrm{Al}$ when doped with boron we attribute to the impurityinduced charge density presented below. These results are consistent with the experimental findings that single crystals show reduced cleavage strength when the boron concentration is more than 0.8 at. $\% .^{10}$ Much larger supercell calculations are required to investigate lower impurity concentrations, and these require substantially increased level of numerical calculations, together with relaxation of the atomic positions, with which we are now engaged and which will be reported elsewhere. ${ }^{22}$

The nonzero values of $s_{0}$ for the $\mathrm{Ni}_{3} \mathrm{AlB}_{1 / 2}$ and $\mathrm{Ni}_{3} \mathrm{AlS}_{1 / 2}$ systems indicate an outward (001) surface relaxation induced by the impurities. By fitting $\Delta E$ to a simple function (cubic polynomial times an exponential) by the nonlinear leastsquares method, we have determined the variation of tensile stress with cleavage separation. The tensile stress, a quantity of central interest for the cleavage calculation, is simply $\sigma=\left(1 / a^{2}\right)(d \Delta E / d s)$, where $a$ is the lattice constant. Figure 2(b) shows the variation of tensile stress with separation between neighboring slabs for pure $\mathrm{Ni}_{3} \mathrm{Al}$ (solid curve), for $\mathrm{Ni}_{3} \mathrm{AlB}_{1 / 2}$ (dotted curve), and for $\mathrm{Ni}_{3} \mathrm{AlS}_{1 / 2}$ (dashed curve). Calculated values for the ideal cleavage energy, the ideal yield stress, and the ideal yield strain for pure $\mathrm{Ni}_{3} \mathrm{Al}$, for $\mathrm{Ni}_{3} \mathrm{AlB}_{1 / 2}$, and for $\mathrm{Ni}_{3} \mathrm{AlS}_{1 / 2}$ are listed in Table I, respectively. We find an ideal cleavage energy of $6.3 \mathrm{~J} \mathrm{~m}^{-2}$ for pure $\mathrm{Ni}_{3} \mathrm{Al}$, which compares well with the value of $5.8 \mathrm{~J} \mathrm{~m}^{-2}$ found by $\mathrm{Fu}$ and Yoo. ${ }^{23}$ It is important to notice, that while boron increases the maximum cleavage stress (ideal yield stress) by about $2 \%$ compared to that of the pure $\mathrm{Ni}_{3} \mathrm{Al}$ system, sulfur causes a significant reduction of $\sigma_{\max }$ by $29 \%$. These results for the contrasting effects of boron and sulfur on the maximum ideal yield stress of $\mathrm{Ni}_{3} \mathrm{Al}$ are similar to those found for these impurities placed interstitially in a nickel cluster. ${ }^{17}$ More recently, we found ${ }^{22}$ that reduction of the impurity (boron or sulfur) concentration by a factor of 2 (5.88 at. \%) produces small changes $(2-5 \%)$ of the maximum yield stress attained at the higher (11.1 at. \%) concentration. It remains to be seen, whether the effects of boron may become more pronounced in nonstoichiometric (nickelrich) $\mathrm{Ni}_{3} \mathrm{Al}$ compounds. ${ }^{24}$

In order to understand the contrasting effects of boron and sulfur on the ideal yield stress of $\mathrm{Ni}_{3} \mathrm{Al}$ in Fig. 2, it is important to study the rearrangement of electronic charge induced by the impurities within and across the cleavage plane. We have calculated the impurity-induced charge density, $\Delta \rho_{\text {ind }}=\rho_{\text {solid }}\left(\mathrm{Ni}_{3} \mathrm{Al} X_{1 / 2}\right)-\rho_{\text {solid }}\left(\mathrm{Ni}_{3} \mathrm{Al}\right)$ $-\rho_{\text {atom }}\left(X_{1 / 2}\right)$. (The first two terms are the self-consistent charge density of the slab with and without the impurity, and the last term is the superposition of atomic charge densities centered on impurity sites). In Figs. 3 and 4 we show the boron- and sulfur-induced charge density on the vertical (200) plane (the plane which contains just nickel atoms and the impurity atom in Fig. 1), respectively, for several values of the slab separation. The solid (dotted) contours represent accumulation (depletion) of electronic charge. We find that there is a large charge accumulation between the boron and $\mathrm{Ni}(4)$ atoms across the cleavage plane for $\mathrm{Ni}_{3} \mathrm{AlB}_{1 / 2}$, while there is a large depletion of charge in the region between the sulfur and the $\mathrm{Ni}(4)$ atom for $\mathrm{Ni}_{3} \mathrm{AlS}_{1 / 2}$. These results are in contrast to those of Messmer and Briant, ${ }^{13}$ where embrittling 
elements draw charge from neighboring metal atoms onto themselves, thereby weakening the metal-metal bond charge network. The strong accumulation of bonding charge which "hangs on" across the interface much longer, explains the fact that the ideal cleavage strength for $\mathrm{Ni}_{3} \mathrm{AlB}_{1 / 2}$ is larger than that of $\mathrm{Ni}_{3} \mathrm{AlS}_{1 / 2}$, and we believe it is the microscopic basis for the resistance of the boron-doped system to cleavage. On the other hand, both boron and sulfur impurities induce an accumulation of interstitial bonding charge between the nearest-neighbor $\mathrm{Ni}(5)$ atoms in the (001) plane, indicating clearly the anisotropic character of the bonding charge and hence the tensorial nature of the forces along and across the cleavage plane. The effect of charge redistribution during the straining process has been suggested to play an important role in determining the material's tensile strength. ${ }^{16}$

In order to gain insight at the microscopic level into the effect of the impurities, we show in Figs. 5(a) and 5(b) the $l$ and site-projected density of states (DOS) for $\mathrm{Ni}_{3} \mathrm{AlB}_{1 / 2}$ and $\mathrm{Ni}_{3} \mathrm{AlS}_{1 / 2}$, respectively, and compare them with those of pure $\mathrm{Ni}_{3} \mathrm{Al}$ (dashed lines) for the slab separation $s_{0}$. The density of states show a strong intra- and interplanar hybridization between the $\mathrm{B} p$ and $\mathrm{Ni}(5) d$ and $\mathrm{Ni}(4) d$ states. As expected, the interaction of the impurity with the $\mathrm{Ni}(3)$ atoms is similar to that with $\mathrm{Ni}(4)$ atoms for small cleavage separations. The boron $p_{x}, p_{y}, p_{z}$ orbitals hybridize with the $\mathrm{Ni} d_{x^{2}-y^{2}}$ and $d_{z^{2}}$ orbitals of $e_{g}$ symmetry forming bonding and antibonding $e_{g}-p$ states. As can be seen clearly in Fig. 3, this hybridization gives rise to a depletion of electron density from the Ni $e_{g}-p$ antibonding state (mainly $d_{x^{2}-y^{2}}$ or $d_{z^{2}}$ orbitals at the $\mathrm{Ni}(5)$ and $\mathrm{Ni}(4)$ sites), accompanied by a charge buildup in the $t_{2 g}$-type antibonding $d$ orbitals pointing along the nearest-neighbor Ni-Ni direction (mainly $d_{x y}, d_{x z}$, and $d_{y z}$ at $\mathrm{Ni}$ sites). In the pure $\mathrm{Ni}_{3} \mathrm{Al}$ system, the $\mathrm{Ni} d$ hole states above the Fermi energy are antibonding states of $t_{2 g}-t_{2 g} \sigma$ type. Thus, the $p$ - $d$ interaction fills the $t_{2 g}$ antibonding states, resulting in a downward shift of the $\mathrm{Ni}(4)$ and $\mathrm{Ni}(5)$ $d$-projected DOS in Fig. 5(a). Similar results for the rigidity of the boron-induced charge density were reported by $\mathrm{Wu}$, Freeman, and Olson for iron grain boundaries ${ }^{18}$ and by Eberhart and Vvedensky for $\mathrm{Ni}_{3} \mathrm{Al}$ grain boundaries. ${ }^{14,15} \mathrm{On}$ the other hand, as can be seen in Fig. 5(b) the overlap between the sulfur $p$ and $\mathrm{Ni}(5) d$ and $\mathrm{Ni}(4) d$ derived bands is smaller in $\mathrm{Ni}_{3} \mathrm{AlS}_{1 / 2}$, indicating a weaker hybridization between the sulfur $p_{x}, p_{y}, p_{z}$, and nickel $d$ orbitals. This is consistent with the depletion of bonding charge in the vicinity of the sulfur atom. Thus, the downward shift of the $\mathrm{Ni}(4)$ and $\mathrm{Ni}(5)$ $d$-projected DOS in Fig. 5(b) appears to be not through hybridization but rather through charge transfer of more ionic character. The downward shifts in the $\mathrm{Ni}(5)$ and $\mathrm{Ni}(4) d$ DOS are consistent with the calculated values of charge transfer for these atoms.

To summarize, we have presented self-consistent totalenergy FLMTO electronic-structure calculations of the role of boron and sulfur impurities on the ideal cleavage fracture properties (ideal cleavage energy, ideal yield stress) of $\mathrm{Ni}_{3} \mathrm{Al}$ under tensile stress. The electronic bonding mechanism underlying the contrasting effects of the boron and sulfur impurities seems to be strong $d$ - $p$ hybridization for the boron impurity and a more embeddedlike (electrostatic) interaction for the sulfur impurity. Work currently in progress is aimed at the effects of boron concentration and of relaxation of the crystalline atomic positions on the ideal cleavage properties. In the near future we intend to proceed with calculations involving the sliding of one half of a crystal relative to another along a slip plane in order to investigate the competition between dislocation emission and cleavage, and calculations which involve the geometry of grain boundaries.

We have benefited greatly from discussions with Dr. Ruqian Wu and Dr. Say Peng Lim. The research at California State University Northridge (CSUN) was supported through the U.S. Army Research Office under Grant No. DAAH0493-G-0427 and the Office of Research and Sponsored Projects at CSUN.
${ }^{1}$ C. T. Liu and D. P. Pope, in Intermetallic CompoundsPrinciples and Practices, edited by J. H. Westbrook and R. L. Fleischer (Wiley, New York, 1995), Vol. 2, p. 17.

${ }^{2}$ G. H. Meier and F. S. Petit, Mater. Sci. Eng. A 153, 548 (1992).

${ }^{3}$ K. Aoki and O. Izumi, J. Jpn. Inst. Metals 43, 1190 (1970).

${ }^{4}$ K. Aoki and O. Izumi, Acta Metall. 27, 807 (1979).

${ }^{5}$ C. T. Liu, in Alloy Phase Stability, edited by A. Gonis and G. M. Stocks (Kluwer, Dordrecht, 1989), p. 7.

${ }^{6}$ C. L. White and D. F. Stein, Metall. Trans. A 9A, 13 (1978).

${ }^{7}$ Takayuki Takasugi, in Intermetallic Compounds-Principles and Practices, edited by J. H. Westbrook and R. L. Fleischer (Wiley, New York, 1995), Vol. 1, p. 585.

${ }^{8}$ P. Johnson, W. Gourdin, A. Gonis, N. Kioussis, and M. D. Vaudin (unpublished).

${ }^{9}$ Clyde Briant, in Intermetallic Compounds-Principles and Practices (Ref. 7), p. 895.

${ }^{10}$ F. E. Heredia and D. P. Pope, Acta Metall. Mater. 39, 2017 (1991).

${ }^{11}$ S. P. Chen, A. F. Voter, R. C. Albers, A. M. Boring, and P. J. Hay, J. Mater. Res. 5, 955 (1990).
${ }^{12}$ J. Rice, J. Mech. Phys. Solids 40, 239 (1992).

${ }^{13}$ R. P. Messmer and C. L. Briant, Acta Metall. 30, 457 (1982).

${ }^{14}$ M. E. Eberhart and D. D. Vvedensky, Phys. Rev. Lett. 58, 61 (1987).

${ }^{15}$ M. E. Eberhart and D. D. Vvedensky, Scr. Metall. 22, 183 (1988).

${ }^{16}$ M. E. Eberhart, D. P. Clougherty, and J. N. Louwen, Mater. Res. Bull. 16, 53 (1991).

${ }^{17}$ G. S. Painter and F. W. Averill, Phys. Rev. Lett. 58, 234 (1987).

${ }^{18}$ R. Wu, A. J. Freeman, and G. B. Olson, Science 265, 376 (1994).

${ }^{19}$ David L. Price and Bernard R. Cooper, Phys. Rev. B 39, 4945 (1989).

${ }^{20}$ David L. Price, John M. Wills, and Bernard R. Cooper, Phys. Rev. B 48, 15301 (1993).

${ }^{21}$ D. Hackenbracht and J. Kubler, J. Phys. F 13, L179 (1983).

${ }^{22}$ N. Kioussis, D. Iotova, S. Sun, and M. Ciftan (unpublished).

${ }^{23}$ C. L. Fu and M. H. Yoo, Mater. Chem. Phys. 32, 32 (1992).

${ }^{24}$ V. Vitek (private communication); J. J. Kruisman, V. Vitek, and J. Th. M. De Hosson, Acta Metall. 36, 2729 (1988); D. M. Dimiduk and T. A. Parthasarathy, Philos. Mag. Lett. 71, 21 (1995). 